\title{
Evaluation of Prognostic Factors that Affect Survival Outcomes of Breast Cancer Patients with Brain Metastases: A Single Institutional Experience
}

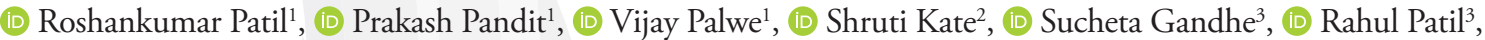 \\ (D) Yasam Venkata Ramesh ${ }^{4}$, (D) Raj Nagarkar ${ }^{5}$ \\ ${ }^{1}$ Department of Radiation Oncology, HCG Manavata Cancer Centre, Maharashtra, India \\ ${ }^{2}$ Department of Medical Oncology, HCG Manavata Cancer Centre, Maharashtra, India \\ ${ }^{3}$ Department of Pathology, HCG Manavata Cancer Centre, Maharashtra, India \\ ${ }^{4}$ Department of Academics, HCG Manavata Cancer Centre, Maharashtra, India \\ ${ }^{5}$ Department of Surgical Oncology, HCG Manavata Cancer Centre, Maharashtra, India
}

\begin{abstract}
Objective: This study aimed to evaluate various prognostic factors that play a vital role in stratifying and guiding tailored treatment strategies and survival outcome in breast cancer patients with brain metastases (BM).

Materials and Methods: Data regarding demography, clinical presentation, molecular subtypes, risk-stratification, treatment details, and outcomes were retrieved from medical records. All time-to-event (survival) outcomes were analyzed by Kaplan-Meir method and compared using log-rank test. Univariate and multivariate analysis of relevant prognostic factors were performed and p-values $\leq 0.05$ were considered statistically significant.

Results: A total of 88 patients (median age: 50 years) were included for this study. The median follow-up time of all surviving patients was -20 months. During the follow-up, $82(93.1 \%)$ patients died. The median survival of all patients was 12 months, with 1-year and 2-year overall survival (OS) rate of $51 \%$ and $22 \%$, respectively. Based on univariate analysis, statistically significant prognostic factors for OS were molecular subtypes, number of BM, and Karnofsky Performance Status (KPS); however, number of BM and KPS emerged as independent predictors of survival based on multivariate analysis.

Conclusion: We conclude that, there are other important prognostic factor, such as number of BM, which may affect the OS of these patients, in addition to variables included in the diagnosis-specific graded prognostic assessment score. Prospective studies evaluating these factors are necessary to further refine the stratification of patients, which will aid the initiation of appropriate treatment to improve the OS of patients.
\end{abstract}

Keywords: Breast cancer, brain metastases, survival outcome, prognostic factors, DS-GPA score

Cite this article as: Patil R, Pandit P, Palwe V, Kate S, Gandhe S, Patil R, Ramesh YV, Nagarkar R. Evaluation of Prognostic Factors that Affect Survival Outcomes of Breast Cancer Patients with Brain Metastases: A Single Institutional Experience. Eur J Breast Health 2021; 17(1): 62-67.

\section{Introduction}

In cancer patients, brain metastases (BM) is among the major causes of morbidity and mortality. It was projected that $-20 \%$ of patients with cancer will develop BM $(1,2)$. The most common cancers associated with BM are breast cancer $(\mathrm{BC})$ colorectal cancer, renal cell cancer, lung cancer, and melanoma. BC is among the most common cancers that cause BM. Approximately 5\%-20\% of metastatic BC patients have BM and, on including autopsy studies, the numbers may increase up to $30 \%(3,4)$. The current National Comprehensive Cancer Network treatment guidelines for BM are based on status of the primary disease and number of metastases. Local treatment involving surgery or stereotactic radiosurgery (SRS) is recommended for patients having few metastases (preferably 1-3 BM) with controlled primary disease. For patients having multiple (>3) BM, Hippocampal Sparing Whole-brain radiotherapy (HS-WBRT) is recommended is the treatment option (5).

Survival of BM patients is very unpredictable and this is due to the tumor biology and patient heterogeneity. However, with advances in technology and systemic therapies, the prognosis of patients and their overall survival can be analyzed and improved using various data-driven prognostic tools including recursive partitioning analysis (RPA) and diagnosis-specific graded prognostic assessment (DS-GPA).

The Radiation Therapy Oncology Group (RTOG) has published the RPA prognostic index for patients with BM (6). The scores in this index are derived based on patient age, Karnofsky Performance Status (KPS), and tumor status (Table 1a). Moreover, this has also been validated by several other studies (7-9). Unfortunately, RTOG-RPA is not specific (in terms of diagnosis) and does not reflect the current 
advances in systemic therapy. To overcome these limitations, GPA was developed, validated, and adapted. In the GPA system, four parameters are evaluated: age, KPS, number of BM, and extra-cranial metastases (ECM). Recently, GPA was updated as DS-GPA index (Table 1b). DS-GPA includes another variable (molecular subtypes of $\mathrm{BC}$ ) as a part of the prognostic factors that determine the overall survival (OS) of patients.

As a result of the heterogeneity of BM, the clinical implications and nuances for management of the treatment differ greatly from patient to patient (10). Therefore, treatments based on a generalized protocol cannot be successful in all patients with BM, thus investigating the need for individualized treatment modalities. Therefore, this study aimed to analyze the survival outcomes and evaluate the factors affecting survival of BC patients with BM.

\section{Materials and Methods}

\section{Patients population}

A total of 88 BC patients (all female, age range: 26-75 years) with BM, who were treated in our centre from Jan 2015 - Dec 2018, were enrolled in this retrospective single-center study. The study was approved by the Institutional Ethics Committee and all participants of this study signed a written informed consent. BM was diagnosed by either computed tomography (CT) head scan or magnetic resonance imaging (MRI) head scan of BC patients with symptoms like headache, vomiting, weakness, dizziness or neurological deficit or any other symptoms of BM. All clinical parameters and outcome data were retrieved from patients' electronic medical records.

All diagnosed cases of BC, as well as radiologically or histologically proven BM patients were included for this study. All BM patients with primaries other than $\mathrm{BC}$ were excluded from the study.

\section{Treatment}

After diagnosis of BM, number of BM was assessed by neuroimaging (MRI scan or CT scan). Patients with multiple BM were treated with
WBRT $(30 \mathrm{~Gy} / 10 \#$ or $20 \mathrm{~Gy} / 5 \#)$, while patients having 1 or $2 \mathrm{BM}$ with controlled primary or extracranial metastasis were treated by SRS. One patient underwent surgical removal of BM, followed by WBRT. After the local treatment for BM, all patients were treated by systemic therapy. OS was defined as the time interval between time of diagnosis of BM and time of death or last follow-up (if the patient is alive).

\section{Prognostic factors for survival}

Univariate and multivariate analyses were used to analyze factors that influence DS-GPA score. The factors considered were age, KPS, number of BM, burden of extra cranial disease, and molecular subtype. Patients were divided into various RPA: Class I (KPS score $\geq 70$ ); II (all patients not at Class I or III), and III (KPS score <70) (Table 1).

\section{Statistical analysis}

The primary endpoint for this analysis was OS time, which was calculated by the Kaplan-Meier method. Statistically comparison of survival distribution was performed by log-rank test [at a significance level ( $p$ value) of $\leq 0.05$ ]. Cox regression model was used to perform univariate and multivariate survival analysis in order to calculate p-value, hazard ratios (HR), and confidence intervals (95\% CI) using SPSS Statistics for Windows Version 22.0 (SPSS, Chicago, IL, USA).

\section{Results}

\section{Clinical profile}

A total of 88 BC patients (median age: 50 years, age range: $26-75$ years, median follow-up of 20 months $=1-56$ months) were enrolled for this study. Patients were classified and distributed according to DS-GPA scoring and RPA class, as shown in Table 1 and 2. In this study, the median KPS score was 70 (range: 40-90). Based on molecular classification, there were 26 Luminal A patients (30\%), 13 Luminal B patients (15\%), 27 HER2 patients (30\%), and 22 patients with Basal-like subtypes (25\%). Of the total (88) patients, various forms of metastasis were observed: BM (15 patients); ECM (73 patients); solitary BM (17 patients); 2-3 BM (05 patients); and multiple metastasis (66 patients). Of the 17 solitary BM patients,

\title{
Table 1a. Recursive partitioning analysis
}

\author{
Class Patient's parameters \\ Class 1 Patients with KPS > or $=70,<65$ years of age with controlled primary and no extra-cranial metastases \\ Class 2 All others \\ Class $3 \quad$ KPS $<70$ \\ KPS: Karnofsky Performance Scale \\ Adapted from: Gaspar et al. ${ }^{6}$
}

Table 1b. Disease Specific Graded Prognostic Index scoring factor

$\begin{array}{llllll}\text { Factors } & 0.0 & 0.5 & 1.0 & 1.5 & 2.0 \\ \text { KPS } & \leq 50 & 60 & 70-80 & 90-100 & - \\ \text { Molecular subtype } & \text { Triple } & - & \text { Luminal A } & \text { HER2 } & \text { Luminal B } \\ \text { negative } & \geq 60 & <60 & - & - & -\end{array}$

Luminal A - HER2 negative ER/PR positive; Luminal B - HER2/ER/PR positive; HER-2 - HER2 positive ER/PR negative; Triple negative - ER/PR/HER2 negative. KPS: Karnofsky Performance Scale; ER: Estrogen receptor; PR: Progesterone Receptor; HER2: Human Epithelial Growth Factor Receptor-2. Adapted from: Sperduto et al. ${ }^{11}$ 
four patients underwent SRS, while three patients underwent surgery as first local treatment, followed by WBRT. The remaining 81 patients completely received WBRT to a dose of 30 Gy in 10 fractions or $20 \mathrm{~Gy}$ in 5 fractions, in view of eliminating or ruling out either multiple BM (>3) or uncontrolled extra-cranial disease (ECD).

\section{Survival analysis}

The primary end point of this analysis was OS time, which was calculated by the Kaplan-Meier method. Median survival of patients was 12 months, while 1 -year and 2-year OS was $51 \%$ and $22 \%$, respectively. As at the time of the study, six patients were alive and 82 patients had died due to the disease.

To calculate the patients' OS using scores, patients were given GPA scores and divided into RPA class according to clinical features (Table 1). The patients score distribution, as well as mean and median survival are given in Table 2 and 3. Results indicate that patients having the highest GPA score of 3.5 had the best survival (16.7 months), while patients with luminal subtypes (15 months) had better survival than patients with non-luminal HER positive (13.6 months) and basal-like group (10.9 months) subtypes. Patients with controlled ECD had better survival when compared to those with uncontrolled ECD. In addition, patients having 1-3 BM lived longer than those having multiple metastases. Patients grouped under RPA class 1-2 were found had a good OS compared to those grouped under RPA class 3 (Table 3).

We performed a detailed univariate analysis of various demographical, clinical, and tumor characteristic factors so as to know their impact on OS of the patients. The log-rank test was used to identify the impact of various factors on OS of the patients. No statistically significant influence was observed with age ( $\leq 60$ years vs $>60$ years, $p=0.51)$ and presence of ECM (Present vs absent, $\mathrm{p}=0.14$ ). However, molecular subtype (luminal vs non-luminal $\mathrm{p}=0.02$ ) (Figure 1 ), number of $\mathrm{BM}$ (single vs multiple $\mathrm{p}=0.002$ ) (Figure 2 ), and performance scale (KPS $\geq 70$ vs $<70 \mathrm{p}=0.021$ ) (Figure 3) showed a statistically significant impact on OS of the patients. Using GPA ( $<2 \mathrm{vs} \geq 2 \mathrm{p}=0.05)$ and RPA class (Class $1-2$ vs class $3 \mathrm{p}=0.02$ ), we also cross-checked the impact of the scores on OS of the patients (Table 4). Patients who had received SRS or underwent surgery had better survival than patients who had received WBRT alone $(\mathrm{p}=0.015)$. This may be due to the higher burden of the disease either in form of multiple BM or uncontrolled ECD in patients who had received WBRT alone.

Table 2. Patients score distribution

\begin{tabular}{|lcc|}
\hline Criteria & Number & Percentage (\%) \\
\hline RPA class & & 12 \\
Class 1 & 10 & 66 \\
Class 2 & 58 & 22 \\
Class 3 & 20 & \\
GPA score & & 12 \\
$0-1$ & 11 & 51 \\
$1.5-2.5$ & 45 & 26 \\
3 & 22 & 11 \\
$3.5-4$ & 10 & \\
\hline
\end{tabular}

64 RPA: Recursive partitioning analysis; GPA: Graded prognostic assessment
Cox proportional hazards model was used to calculate HR and 95\% CI of the multivariate survival analysis. Based on this analysis, only two variables, including KPS (HR: 1.83; 95\% CI: 1.01-3.34; $\mathrm{p}=0.04$ ) and number of BM (HR: 2.48; 95\% CI: 1.18-5.21; $\mathrm{p}=0.01$ ), were found to be independent prognostic factors that affect OS of the patients when compared to others, as shown in Table 5.

\section{Discussion and Conclusion}

At present, various local and systemic treatment modalities are applied for metastatic BC patients to improve their OS. Most importantly, the present need is to identify the most reliable predictors for these BC patient's subset, which will further assist the doctors in initiating

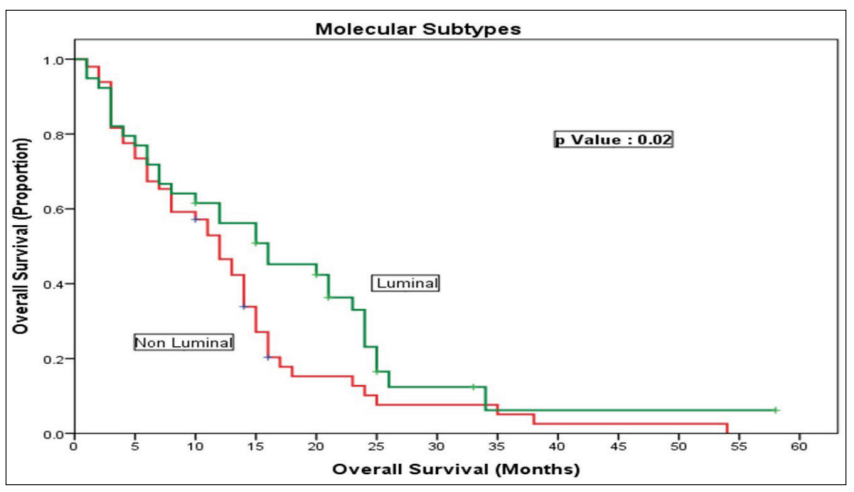

Figure 1. Kaplan-Meier curves of overall survival stratified by molecular subtype

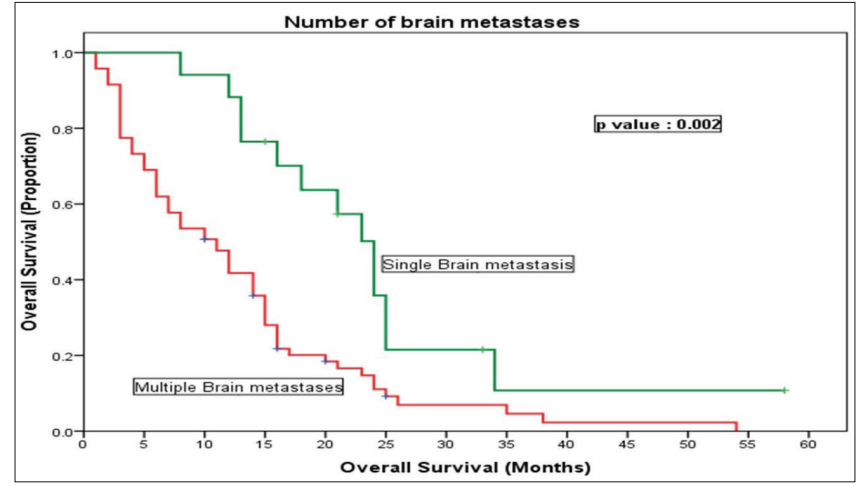

Figure 2. Kaplan-Meier curves of overall survival stratified by number of brain metastases

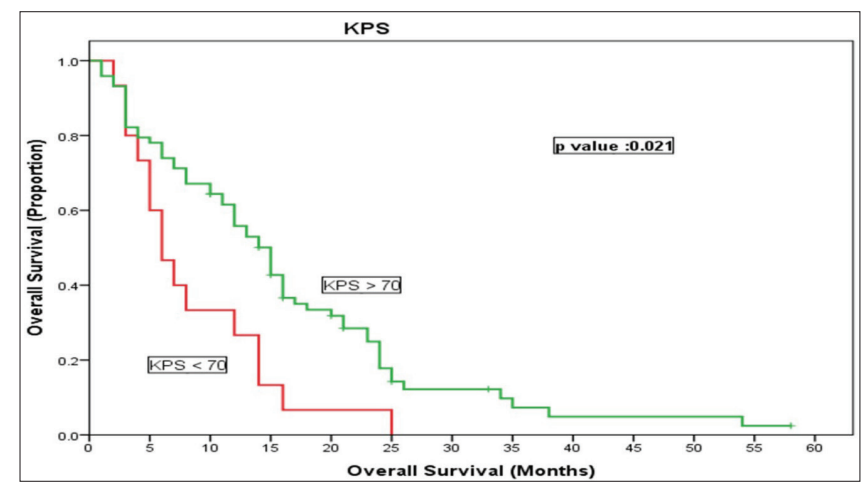

Figure 3. Kaplan-Meier curves of overall survival stratified by KPS KPS: Karnofsky Performance Status 
aggressive treatments immediately. The present study aimed to determine these prognostic factors. Based on the results of the univariate and multivariate analysis, number of BM and KPS emerged as statistically significant prognostic factors $(\mathrm{p}<0.05)$ that affect OS of the patients.

Sperduto et al. (11) reported that median survival of this patient subset is directly proportional to their DS-GPA scores; in this study, we also observed a higher survival of patients with a higher GPA score as compared with patients with a lower GPA score.

Age is considered among the common prognostic factors in GPA scoring, such that patients below 60 years of age are scored 0.5 and patients above 60 years are scored 0 , thus indicating that the former set of the populations had better survival than the latter group. However, from our results, age was found to be an insignificant prognostic factor that affects OS of the patients. This can be explained by the large percentage of triple-negative BC patients below 60 years in the Asian population as compared to those in western population, which itself is a negative prognostic factor in reducing the importance of age (12).

\section{Table 3. Mean and median survival of patients according to prognostic factors}

\begin{tabular}{|c|c|c|}
\hline Variables & $\begin{array}{l}\text { Means survival } \\
\text { (Months) }\end{array}$ & $\begin{array}{l}\text { Median survival } \\
\text { (Months) }\end{array}$ \\
\hline \multicolumn{3}{|l|}{ GPA score } \\
\hline 3.5 & 16.7 & 17.5 \\
\hline 3 & 15.7 & 15 \\
\hline 2.5 & 11.2 & 10 \\
\hline$\leq 2$ & 10 & 8 \\
\hline \multicolumn{3}{|c|}{ Molecular Classification } \\
\hline Luminal A & 15 & 12 \\
\hline Luminal B & 15 & 17 \\
\hline HER2 Rich & 13.6 & 13 \\
\hline Basal Like & 10.9 & 8 \\
\hline \multicolumn{3}{|l|}{ Local Treatment } \\
\hline SRS / Surgery \pm WBRT & 25 & 15 \\
\hline WBRT Only & 10 & 12 \\
\hline \multicolumn{3}{|c|}{ Extra cranial metastases } \\
\hline Present & 12 & 12 \\
\hline Absent & 18 & 15 \\
\hline \multicolumn{3}{|l|}{ RPA Class } \\
\hline Class 1-2 & 15 & 13 \\
\hline Class 3 & 10 & 7 \\
\hline \multicolumn{3}{|c|}{ Number of brain metastases } \\
\hline 1 & 22 & 21 \\
\hline $2-3$ & 12 & 14 \\
\hline$>3$ & 11 & 10 \\
\hline
\end{tabular}

SRS: Stereotactic radiosurgery; WBRT: Whole brain radiotherapy; GPA: Graded prognostic assessment; RPA: Recursive partitioning analysis; HER2: Human epidermal growth factor receptor-2
KPS performance scale is another important prognostic factor that affects survival. Patients having KPS $\geq 70$ had better survival when compared to patients having KPS <70 (HR: 0.56; CI: 95\% $0.33-$ $0.94 ; \mathrm{p}=0.037)$ and this is in agreement with previous studies $(11,13)$. Lower scores indicate the seriousness of the illness and, in many cases, it worsens patients' OS with time $(13,14)$.

Another most important prognostic factor in management and survival of $\mathrm{BC}$ patients is molecular subtypes. Prevalence of molecular subtypes differs in Asian population (Luminal A=37\%, Luminal B=8\%, HER2 Rich $=11 \%$, and Basal-Like $=26 \%$ ) when compared to Western population (Luminal $\mathrm{A}=71 \%$, Luminal $\mathrm{B}=6 \%$, Her-2 Rich $=7 \%$, and Basal-Like=15\%) (15-17). Molecular subtypes always differ in terms of survival. Patients in the triple-negative BC group are worse in terms of survival when compared to ER/PR positive patients (luminal subgroups) $(11,18,19)$. Results from our study on the overall impact on survival (HR: 0.66; CI: 95\% 0.41-1.04; $\mathrm{p}=0.05$ ) are in agreement with those of previous reports $(11,16,18,19)$.

Some studies did not consider the number of BM as an independent prognostic factor (20). Results from our study suggest that patients having single BM had better survival when compare to patients with multiple BM. Based on multivariate analysis, number of BM was found to be an independent prognostic factor (HR: 2.48; CI: 95\% 1.18 $5.21 ; \mathrm{p}=0.01$ ). Considering the impact of number of BM on survival, the removal of this prognostic factor from DS-GPA is questionable.

By considering different prognostic factors and finding patients of likely longer survival, treatments like SRS or surgery can be recommended. Offering such treatments can not only increase the survival of patients, but also decrease the chances of cognitive dysfunctions. Patients not feasible for SRS or surgery can be provided with hippocampus sparing

Table 4. Univariate analysis of prognostic factors that affect survival outcomes $p$-values $\leq 0.05$ are considered statistically significant

\begin{tabular}{lccc} 
Prognostic factors & Variables & 1-year OS & $\begin{array}{c}\text { Log-rank } \\
\text { p-value }\end{array}$ \\
\hline Age & $\leq 60$ years & $54 \%$ & 0.51 \\
& $>60$ years & $48 \%$ & \\
Molecular subtypes & Luminal & $61 \%$ & 0.02 \\
Number of brain & Non luminal & $44 \%$ & \\
mets & Multiple & $76 \%$ & 0.002 \\
KPS & $>70$ & $52 \%$ & \\
Extra cranial & $<70$ & $38 \%$ & 0.02 \\
metastases & Present & $52 \%$ & 0.14 \\
GPA score & Absent & $47 \%$ & \\
& $>2$ & $57 \%$ & 0.05 \\
RPA class & $<2$ & $45 \%$ & \\
\hline
\end{tabular}

OS: Overall survival; KPS: Karnofsky Performance Status; GPA: Graded prognostic assessment; RPA: Recursive partitioning analysis 


\section{Table 5. Multivariate analysis of prognostic factors that affect survival outcomes}

\begin{tabular}{|c|c|c|c|c|}
\hline \multirow[t]{2}{*}{ Prognostic factors } & \multirow[t]{2}{*}{ Variables } & \multicolumn{3}{|c|}{ Overall survival } \\
\hline & & HR & $95 \% \mathrm{Cl}$ & p-value \\
\hline \multirow{2}{*}{ Age } & $<60$ years & \multirow{2}{*}{0.75} & \multirow{2}{*}{$0.39-1.43$} & \multirow{2}{*}{0.38} \\
\hline & $>60$ years (ref) & & & \\
\hline \multirow{2}{*}{ Molecular subtypes } & Luminal & \multirow{2}{*}{0.80} & \multirow{2}{*}{$0.49-1.31$} & \multirow{2}{*}{0.47} \\
\hline & Non luminal (ref) & & & \\
\hline \multirow{2}{*}{ Number of brain mets } & Single (ref) & \multirow{2}{*}{2.48} & \multirow{2}{*}{$1.18-5.21$} & \multirow{2}{*}{0.01} \\
\hline & Multiple & & & \\
\hline \multirow{2}{*}{ KPS } & >70 (ref) & \multirow{2}{*}{1.83} & \multirow{2}{*}{$1.01-3.34$} & \multirow{2}{*}{0.04} \\
\hline & $<70$ & & & \\
\hline \multirow{2}{*}{ Extra cranial mets } & Present (ref) & \multirow{2}{*}{0.89} & \multirow{2}{*}{$0.43-1.82$} & \multirow{2}{*}{0.74} \\
\hline & Absent & & & \\
\hline
\end{tabular}

(HS)-WBRT using intensity-modulated radiotherapy technique. HSWBRT has yielded better results in preserving cognitive functions when compared to WBRT (21). Using HS-WBRT also prevents further decline in cognitive functions in terms of memory or quality of life. Patients who are eligible for this kind of focused treatment should be assessed for likely survival, for which all the prognostic factors mentioned in our present study can be employed.

The major strength of this study is that it is a single institutional study, where all patients were treated using a uniform protocol. This is one among the very few papers that focus on survival of BM patients in Asian population by considering similar variables, classifications, and scoring systems adopted in the Western settings.

Limitations of this study include the retrospective nature of the study, which may impact the overall data. Secondly, there is no detailed follow-up data on treatment protocol before and after the diagnosis of BM. In addition, some of the patients failed to complete the systemic treatment due to social or financial reasons, thus resulting in a comparative lower survival than expected.

In conclusion, we conclude that, there are various other prognostic factors (such as number of brain metastases) other than variables included in the DS-GPA score, which may affect the OS of these patients. Prospective studies evaluating these factors will further refine the stratification of patients, which will aid to initiate of appropriate treatment for improvement of OS of the patients.

\section{Key Points}

- $\quad$ Single institutional experience of clinical outcomes in 88 breast cancer patients with brain metastases and inclusion of molecular classification.

- Acceptable outcomes of 1-year and 2-year overall survival were $51.0 \%$ and $22 \%$, respectively

- Number of brain metastases and KPS emerged as independent predictors of survival based on multivariate analysis.
Acknowledgements: We would like to thank Dr. Yasam Venkata Ramesh from HCG Manavata Cancer Centre, Centre of Difficult Cancers, Nashik, India, for his medical writing assistance.

Ethics Committee Approval: Ethics committee approval was received for this study from the Ethics Committee of Manavata Clinical Research Institute (no: BRBM-1246, date: 15/12/2019).

Informed Consent: Written informed consent was obtained from patients who participated in this study.

Peer-review: Externally peer-reviewed.

\section{Author Contributions}

Concept: R.P, P.P., V.P., R.N.; Design: R.P.; Supervision: P.P., V.P., S.K., S.G., Ra.P., R.N.; Materials: S.K., S.G., Ra.P., R.N.; Data Collection and/or Processing: R.P., P.P., V.P., S.K., S.G., Ra.P.; Analysis and/or Interpretation: R.P., P.P.; Literature Review: R.P., Y.V.R; Writing: R.P., P.P., V.P., Y.V.R.; Critical Review: P.P., V.P., Y.V.R., R.N.

Conflict of Interest: No conflict of interest was declared by the authors.

Financial Disclosure: The authors declared that this study received no financial support.

\section{References}

1. Jemal A, Siegel R, Ward E, Hao Y, Xu J, Murray T, et al. Cancer statistics, 2008. CA Cancer J Clin 2008; 58: 71-96. (PMID: 18287387) [Crossref]

2. Zimm S, Wampler GL, Stablein D, Hazra T, Young HF. Intracerebral metastases in solid-tumor patients: Natural history and results of treatment. Cancer 1981; 48: 384-394. (PMID: 7237407) [Crossref]

3. Kirsch DG, Loeffler JS. Brain metastases in patients with breast cancer: new horizons. Clin Breast Cancer 2005; 6: 115-124. (PMID: 16001989) [Crossref]

4. Tsukada Y, Fouad A, Pickren JW, Lane WW. Central nervous system metastasis from breast carcinoma. Autopsy study. Cancer 1983; 52: 23492354. (PMID: 6640506) [Crossref]

5. Yamamoto M, Serizawa T, Shuto T, Akabane A, Higuchi Y, Kawagishi J, et al. Stereotactic radiosurgery for patients with multiple brain metastases 
(JLGK0901): a multi-institutional prospective observational study. Lancet Oncol 2014; 15: 387-395. (PMID: 24621620) [Crossref]

6. Gaspar L, Scott C, Rotman M, Asbell S, Phillips T, Wasserman T, et al. Recursive partitioning analysis (RPA) of prognostic factors in three Radiation Therapy Oncology Group (RTOG) brain metastases trials. Int J Radiat Oncol Biol Phys 1997; 37: 745-751. (PMID: 9128946) [Crossref]

7. Nieder C, Nestle U, Motaref B, Walter K, Niewald M, Schnabel K. Prognostic factors in brain metastases: should patients be selected for aggressive treatment according to recursive partitioning analysis (RPA) classes? Int J Radiat Oncol Biol Phys 2000; 46: 297-302. (PMID: 10661335) [Crossref]

8. Tendulkar RD, Liu SW, Barnett GH, Vogelbaum MA, Toms SA, Jin T, et al. RPA classification has prognostic significance for surgically resected single brain metastasis. Int J Radiat Oncol Biol Phys 2006; 66: 810-817. (PMID: 17011454) [Crossref]

9. Cannady SB, Cavanaugh KA, Lee SY, Bukowski RM, Olencki TE, Stevens $\mathrm{GH}$, et al. Results of whole brain radiotherapy and recursive partitioning analysis in patients with brain metastases from renal cell carcinoma: a retrospective study. Int J Radiat Oncol Biol Phys 2004; 58: 253-258. (PMID: 14697446) [Crossref]

10. Sperduto PW, Kased N, Roberge D, Xu Z, Shanley R, Luo X, et al. Summary report on the graded prognostic assessment: an accurate and facile diagnosis-specific tool to estimate survival for patients with brain metastases. J Clin Oncol 2012; 30: 419-425. (PMID: 22203767) [Crossref]

11. Sperduto PW, Kased N, Roberge D, Xu Z, Shanley R, Luo X, et al. Effect of tumor subtype on survival and the graded prognostic assessment for patients with breast cancer and brain metastases. Int J Radiat Oncol Biol Phys 2012; 82: 2111-2117. (PMID: 21497451) [Crossref]

12. Triple-Negative Breast Cancer. Last Accessed Date: 07.02.2020. Available from: https://www.breastcancer.org/symptoms/diagnosis/trip_neg.

13. Mirza P, Miriam HAB, Markus S, Christopher N, Barbara C. Retrospective study of 229 surgically treated patients with brain metastases: Prognostic factors, outcome and comparison of recursive partitioning analysis and diagnosis-specific graded prognostic assessment. Surg Neurol Int 2017; 8: 259. (PMID: 29184710) [Crossref]

14. Villà S, Weber, DC, Moretones C, Anabel M, Christophe C, Josep J, et al. Validation of the new graded prognostic assessment scale for brain metastases: a multicenter prospective study. Radiat Oncol 2011; 6: 23. (PMID: 21366924) [Crossref]

15. Pandit P, Patil R, Palwe V, Gandhe S, Patil R, Nagarkar R. Prevalence of Molecular Subtypes of Breast Cancer: A Single Institutional Experience of 2062 Patients. Eur J Breast Health 2019; 16: 39-43. (PMID: 31912012) [Crossref]

16. Onitilo AA, Engel JM, Greenlee RT, Mukesh BN. Breast cancer subtypes based on er/pr and her2 expression: comparison of clinicopathologic features and survival. Clin Med Res 2009; 7(1-2) :4-13. (PMID: 19574486) [Crossref]

17. Mid-Atlantic Division of the Cooperative Human Tissue Network. Last Accessed Date: 07.02.2020. Available from: URL: http://www.cdp.nci. nih.gov/breast/prognostic_dm.html.

18. Lim YJ, Lee SW, Choi N, Kwon J, Eom KY, Kang E, et al. Failure patterns according to molecular subtype in patients with invasive breast cancer following postoperative adjuvant radiotherapy: long-term outcomes in contemporary clinical practice. Breast Cancer Res Treat 2017; 163: 555563. (PMID: 28315066) [Crossref]

19. Shen Q, Sahin AA, Hess KR, Suki D, Aldape KD, Sawaya R, et al. Breast cancer with brain metastases: clinicopathologic features, survival, and paired biomarker analysis. Oncologist 2015; 20: 466-473. (PMID: 25802405) [Crossref]

20. Ahn HK, Lee S, Park YH, Sohn JH, Jo JC, Ahn JH, et al. Prediction of outcomes for patients with brain parenchymal metastases from breast cancer (BC): a new BC-specific prognostic model and a nomogram. Neuro Oncol 2012; 14: 1105-1113. (PMID: 22693244) [Crossref]

21. Gondi V, Pugh SL, Tome WA, Caine C, Corn B, Kanner A, et al. Preservation of memory with conformal avoidance of the hippocampal neural stem-cell compartment during whole-brain radiotherapy for brain metastases (RTOG 0933): a phase II multi-institutional trial. J Clin Oncol 2014; 32: 3810-3816. (PMID: 25349290) [Crossref] 